Article

\title{
Application of Fractional Residual Power Series Algorithm to Solve Newell-Whitehead-Segel Equation of Fractional Order
}

\author{
Rania Saadeh ${ }^{1}$, Mohammad Alaroud ${ }^{2}$, Mohammed Al-Smadi ${ }^{3}$ (i), Rokiah Rozita Ahmad ${ }^{2, *(1)}$ \\ and Ummul Khair Salma Din ${ }^{2}$ \\ 1 Department of Mathematics, Faculty of Science, Zarqa University, Zarqa 13110, Jordan; rsaadeh@zu.edu.jo \\ 2 Center for Modeling and Data Science, Faculty of Science and Technology, Universiti Kebangsaan Malaysia, \\ 43600 Bangi, Selangor DE, Malaysia; mohammadaloroud@yahoo.com (M.A.); \\ ummul@ukm.edu.my (U.K.S.D.) \\ 3 Department of Applied Science, Ajloun College, Al-Balqa Applied University, Ajloun 26816, Jordan; \\ mhm.smadi@bau.edu.jo \\ * Correspondence: rozy@ukm.edu.my; Tel.: +60-3-8921-3716
}

Received: 18 October 2019; Accepted: 9 November 2019; Published: 20 November 2019

\begin{abstract}
The Newell-Whitehead-Segel equation is one of the most nonlinear amplitude equations that plays a significant role in the modeling of various physical phenomena arising in fluid mechanics, solid-state physics, optics, plasma physics, dispersion, and convection system. In this analysis, a recent numeric-analytic technique, called the fractional residual power series (FRPS) approach, was successfully employed in obtaining effective approximate solutions to the Newell-Whitehead-Segel equation of the fractional sense. The proposed algorithm relies on a generalized classical power series under the Caputo sense and the concept of an error function that systematically produces an analytical solution in a convergent fractional power series form with accurately computable structures, without the need for any unphysical restrictive assumptions. Meanwhile, two illustrative applications are included to show the efficiency, reliability, and performance of the proposed technique. Plotted and numerical results indicated the compatibility between the exact and approximate solution obtained by the proposed technique. Furthermore, the solution behavior indicates that increasing the fractional parameter changes the nature of the solution with a smooth sense symmetrical to the integer-order state.
\end{abstract}

Keywords: fractional Newell-Whitehead-Segel model; Caputo fractional derivative; fractional residual power series algorithm; multiple fractional power series

\section{Introduction}

The subject of fractional calculus (FC) is not new, it dates back to the late seventeenth century. It is a generalization of classic calculus that deals with the ordinary differentiation and integration of arbitrary order. Recently, FC has been applied by many researchers to formulate many nonlinear problems that occur in physics and applied mathematics, and it already provides an excellent tool to describe the hereditary and memory properties of these physical phenomena. However, mathematical modeling of real-world problems often leads to nonlinear partial differential equations (NPDEs), including nonlinear oscillations of earthquakes, nonlinear optics, viscoelasticity, fluid flow, chemical reactions, fluid-dynamics traffic, and control theory [1-6]. 
The current work aims to provide a convenient way to ascertain the convergence of approximation series solutions. To achieve our aim, consider the fractional Newell-Whitehead-Segel equation (FNWSE) in the following form:

$$
\mathcal{D}_{t}^{\beta} \varphi(x, t)=a \mathcal{D}_{x}^{2} \varphi(x, t)+b \varphi(x, t)-c \varphi^{p}(t, x), t \geq 0, x \in \mathbb{R}, 0<\beta \leq 1,
$$

subject to the initial condition

$$
\varphi(x, 0)=h_{0}(x),
$$

where $\mathcal{D}_{t}^{\beta}=\frac{\partial^{\beta}}{\partial t^{\beta}}, \mathcal{D}_{x}^{2}=\frac{\partial^{2}}{\partial x^{2}}, a, b, c$ are real constants such that $a, c>0$, and $p \in \mathbb{Z}^{+}$. The term $\mathcal{D}_{t}^{\beta} \varphi(x, t)$ represents the variation of $\varphi(x, t)$ with respect to temporal variable $t$ at a set position, while $\mathcal{D}_{x}^{2} \varphi(x, t)$ expresses the variation of $\varphi(x, t)$ with respect to spatial variable $x$ at a specific time $t$, and $b \varphi(x, t)-c \varphi^{p}(t, x)$ expresses a nonlinear source term for $p>1$. Here, the unknown function $\varphi(x, t)$ can be assumed to be the nonlinear distribution of temperature in a thin and infinitely long rod. It may also be considered as the velocity of fluid flow in a tube of unlimited length with a small diameter.

The classic Newell-Whitehead-Segel model (NWS) is one of the most popular amplitude equations describing the occurrence of stationary spatial stripe patterns in a two-dimensional system as well as the dynamic behavior near the bifurcation point of the Rayleigh-Benard convection of binary fluid mixtures [7]. Indeed, two types of patterns can be observed: the roll pattern, in which the cylinders are formed using fluid stream lines that may be bent and form spirals like patterns, and the hexagonal pattern, in which the honey comb and stripes cells are formed by dividing the flow of liquid. For instance, the patterns of stripes can be found in the visual cortex, on zebra skin, and in human fingerprints. It is worth mentioning that the hexagonal patterns can be achieved in a chemical reaction and diffusion model by utilizing laser beam propagation through nonlinear optical media [8].

Numerous mathematical formations contain nonlinear fractional partial differential equations FPDEs. However, solving these equations is usually difficult. Therefore, effective and developed algorithms are needed to obtain analytical or approximate solutions to these equations. Recently, many common numeric-analytic techniques have been proposed for solving the FNWSE such as the homotopy analysis sumudu transform method [9], variational iteration method [10], Adomian decomposition method [11], and fractional complex transform method [12]. For further research papers regarding numerical techniques for fractional ordinary and partial differential equations arising in different branches of science, we refer to [13-18].

The objective of this work is to apply an advanced algorithm, called the fractional residual power series (FRPS) algorithm, for solving the time-FNWSE. The FRPS is a novel numeric-analytic technique for dealing with both linear and nonlinear issues, which enables us to obtain analytical and approximate solutions in convergent fractional power series (FPS) by combining Taylor's fractional series formula and residual error functions without requiring any constrained assumptions [19-23]. The outline of this work is organized as follows. In Section 2, fundamental definitions and theorems about fractional calculus and FPS are shown. The solution methodology of the FRPS algorithm is introduced in Section 3. In Section 4, some numerical applications are included to clarify the accuracy, simplicity, and reliability of the proposed algorithm. Finally, Section 5 is devoted to conclusions.

\section{Preliminaries and Notations}

In the present section, we recalled some basic definitions and properties of the Caputo and Riemann-Liouville fractional operators. Then, we survey the most important definitions and results related to the fractional Taylor series representation.

Definition 1. [1] A real function $\varphi(t), t>0$ is said to be in the space $C_{\delta}, \delta \in \mathbb{R}$, if there exists a real number $v>\delta$, such that $\varphi(t)=t^{v} \cdot \varphi_{1}(t)$, where $\varphi_{1}(t) \in C[0, \infty)$ and it is said to be in the space $C_{\delta}^{n}$ if $\varphi^{(n)}(t) \in C_{\delta}, n \in \mathbb{N} \cup\{0\}$. 
Definition 2. [1] The Riemann-Liouville fractional integral operator of order $\beta \geq 0$, for a function $\varphi \in C_{\delta}$, $\delta>-1$, is given by:

$$
\mathcal{J}_{t}^{\beta} \varphi(t)=\left\{\begin{array}{ll}
\frac{1}{\Gamma(\beta)} \int_{s}^{t} \frac{\varphi(\eta)}{(t-\eta)^{1-\beta}} d \eta, & 0 \leq s<\eta<t, \beta>0 \\
\varphi(t), & \beta=0
\end{array} .\right.
$$

Definition 3. [1] The Caputo fractional derivative operator of order $\beta>0$ for a function $\in C_{-1}^{n}, n \in \mathbb{N}$, is given by:

$$
\mathcal{D}_{t}^{\beta} \varphi(t)=\left\{\begin{array}{cl}
\frac{1}{\Gamma(n-\beta)} \int_{s}^{t} \varphi^{(n)}(\eta)(t-\eta)^{n-\beta-1} d \eta, & t>\eta>s \geq 0, n-1<\beta<n \\
\frac{d^{n}}{d t^{n}} \varphi(t), & \beta=n \in \mathbb{N}
\end{array} .\right.
$$

$\mathcal{D}_{t}^{\beta}$, we have the following properties:

1. $\mathcal{D}_{t}^{\beta} c=0, c \in \mathbb{R}$.

2. $\mathcal{D}_{t}^{\beta} t^{q}=\frac{\Gamma(q+1)}{\Gamma(q+1-\beta)} t^{q-\beta}, n-1<\beta \leq n, q>n-1, n \in \mathbb{N}, q \in \mathbb{R}$.

3. $\mathcal{D}_{t}^{\beta}\left(\lambda \varphi_{1}(t)+\mu \varphi_{2}(t)\right)=\lambda \mathcal{D}_{t}^{\beta} \varphi_{1}(t)+\mu \mathcal{D}_{t}^{\beta} \varphi_{2}(t)$, that is, $\mathcal{D}_{t}^{\beta}$ is a linear, $\lambda, \mu \in \mathbb{R}$.

Definition 4. [5] A fractional power series (FPS), where $0 \leq n-1<\beta \leq n, x \in I$ and $t \geq t_{0}$,

$$
\sum_{j=0}^{\infty} h_{j}(x)\left(t-t_{0}\right)^{j \beta}=h_{0}(x)+h_{1}(x)\left(t-t_{0}\right)^{\beta}+h_{2}(x)\left(t-t_{0}\right)^{2 \beta}+\ldots,
$$

is called multiple fractional power series (MFPS) about $t_{0}$.

Theorem 1. [5] Suppose that $\varphi(x, t)$ has the following MFPS representation at $t=t_{0}$ :

$$
\varphi(x, t)=\sum_{j=0}^{\infty} h_{j}(x)\left(t-t_{0}\right)^{j \beta}, 0 \leq n-1<\beta \leq n, x \in I, t_{0} \leq t<t_{0}+R .
$$

where $\varphi(x, t)$ is continuous on $I \times\left[t_{0}, t_{0}+R^{1 / \beta}\right)$ and $\mathcal{D}_{t}^{j \beta} \varphi(x, t)$ are continuous on $I \times\left(t_{0}, t_{0}+R^{1 / \beta}\right)$, $j=0,1,2, \ldots$, and $\mathcal{D}_{t}^{k \beta} \mathcal{D}_{t}^{j \beta} \varphi(x, t)$ is well defined on $\left(t_{0}, t_{0}+R^{1 / \beta}\right)$ for $k=1,2, \ldots, n-1$ and $j=0,1,2, \ldots$, then the coefficients $h_{j}(x)$ will be in the form $h_{j}(x)=\frac{\mathcal{D}_{t}^{j \beta} \varphi\left(x, t_{0}\right)}{\Gamma(j \beta+1)}$ such that $\mathcal{D}_{t}^{j \beta}=\mathcal{D}_{t}^{\beta} \cdot \mathcal{D}_{t}^{\beta} \cdots \mathcal{D}_{t}^{\beta}$ (j times).

Proof. Assuming that $\varphi(x, t)=h_{0}(x)+h_{1}(x)\left(t-t_{0}\right)^{\beta}+h_{2}(x)\left(t-t_{0}\right)^{2 \beta}+\ldots, t_{0} \leq t<t_{0}+R^{1 / \beta}$, then directly $h_{0}(x)=\varphi\left(x, t_{0}\right)$. By applying $\mathcal{D}_{t}^{\beta}$ to $\varphi(x, t)$ and evaluating the result at $t_{0}$, it yields that $\mathcal{D}_{t}^{\beta} \varphi\left(x, t_{0}\right)=\Gamma(\beta+1) h_{1}(x)$ and hence $h_{1}(x)=\frac{\mathcal{D}_{t}^{\beta} \varphi\left(x, t_{0}\right)}{\Gamma(\beta+1)}$. Proceeding inductively and applying $\mathcal{D}_{\mathrm{t}}^{j \beta}$ to $\varphi(x, t) j$ times and evaluating the result at $t_{0}$, one can note that $\mathcal{D}_{t}^{j \beta} \varphi\left(x, t_{0}\right)=\Gamma(j \beta+1) h_{j}(x)$ and hence the proof is completed.

Theorem 2. [5] Let $\beta \in(n-1, n]$ and suppose that $(x, t) \in C\left[t_{0}, t_{0}+R^{1 / \beta}\right), \mathcal{D}_{t}^{j \beta} \varphi(x, t) \in C\left(t_{0}, t_{0}+R^{1 / \beta}\right)$, and $\mathcal{D}_{t}^{k \beta} \mathcal{D}_{t}^{j \beta} \varphi(x, t)$ is well defined on $\left(t_{0}, t_{0}+R^{1 / \beta}\right)$ for $k=1,2, \ldots, n-1$, and $j=0,1,2, \ldots$. Then,

$$
\mathcal{J}_{t}^{(m+1) \beta} \mathcal{D}_{t}^{(m+1) \beta} \varphi(x, t)=\varphi(x, t)-\sum_{j=0}^{m} \frac{\mathcal{D}_{t}^{j \beta} \varphi\left(x, t_{0}\right)}{\Gamma(j \beta+1)}\left(t-t_{0}\right)^{j \beta} .
$$

It is worth mentioning here that the MFPS representation about $t=t_{0}$ can be rewritten by

$$
\varphi(x, t)=\varphi_{m}(x, t)+\mathcal{R}_{m}(x, t),
$$


where $\varphi_{m}(x, t)$ represents the mth approximate series of $\varphi(x, t)$ and $\mathcal{R}_{m}(x, t)$ is the reminder term of MFPS, which are given, respectively, by

$$
\varphi_{m}(x, t)=\sum_{j=0}^{m} h_{j}(x)\left(t-t_{0}\right)^{j \beta},
$$

and $\mathcal{R}_{m}(x, t)=\mathcal{J}_{t}^{(m+1) \beta} \mathcal{D}_{t}^{(m+1) \beta} \varphi(x, t)$, with $h_{j}(x)=\frac{\mathcal{D}_{t}^{j \beta} \varphi\left(x, t_{0}\right)}{\Gamma(j \beta+1)}, j=0,1, \ldots, n$. Thus, the MFPS is convergent whenever $\lim _{m \rightarrow \infty} \mathcal{R}_{m}(x, t)=0$.

\section{Solution Methodology of the FRPS Algorithm}

The main objective of this section is to describe the methodology of the proposed algorithm for obtaining the MFPS solution for the FNWSEs (1) and (2) through substituting the expansion of their MFPS among their truncated residual functions.

According to the FRPS approach, let the solution of FNWSEs (1) and (2) about $t_{0}=0$, have the following MFPS:

$$
\varphi(x, t)=\sum_{n=0}^{\infty} h_{n}(x) \frac{t^{n \beta}}{\Gamma(n \beta+1)}, 0<\beta \leq 1, t \geq 0, x \in \mathbb{R},
$$

and let $\varphi_{k}(x, t)$ be the $k$ th truncated series of $\varphi(x, t)$ such that

$$
\varphi_{k}(x, t)=\sum_{n=0}^{k} h_{n}(x) \frac{t^{n \beta}}{\Gamma(n \beta+1)} .
$$

Using the initial condition (2) in expansion (3), we find that $\varphi(x, 0)=h_{0}(x)$. Thus, the MFPS solution (4) can be written as:

$$
\varphi_{k}(x, t)=h_{0}(x)+\sum_{n=1}^{k} h_{n}(x) \frac{t^{n \beta}}{\Gamma(n \beta+1)},
$$

where the coefficients $h_{n}(x), n=1,2,3, \ldots k$, can be determined by solving the following fractional differential equation:

$$
\mathcal{D}_{t}^{(k-1) \beta} \operatorname{Res}_{\varphi}^{k}(x, t)_{t=0}=0, k=1,2,3, \ldots,
$$

in which $\operatorname{Res}_{\varphi}^{k}$ is called the $k$ th residual function and defined as:

$$
\operatorname{Res}_{\varphi}^{k}(x, t)=\mathcal{D}_{t}^{\beta} \varphi_{k}(x, t)-a \mathcal{D}_{x}^{2} \varphi_{k}(x, t)-b \varphi_{k}(x, t)+c \varphi_{k}^{p}(t, x),
$$

whereas the residual function $\operatorname{Res}_{\varphi}$ is defined as:

$$
\operatorname{Res}_{\varphi}(x, t)=\lim _{k \rightarrow \infty} \operatorname{Res}_{\varphi}^{k}(x, t)=\mathcal{D}_{t}^{\beta} \varphi(x, t)-a \mathcal{D}_{x}^{2} \varphi(x, t)-b \varphi(x, t)+c \varphi^{p}(t, x) .
$$

Evidently, $\operatorname{Res}_{\varphi}(x, t)=0$ and $\lim _{k \rightarrow \infty} \operatorname{Res}_{\varphi}^{k}(x, t)=\operatorname{Res}_{\varphi}(x, t)=0$ for each $x \in \mathbb{R}$ and $0<t<\rho$, where $\rho$ is called the convergence radius for the MFPS (3). In [24-28], it has been proved that $\mathcal{D}_{t}^{j \beta} \operatorname{Res}_{\varphi}(x, t)=0$. Also, $\mathcal{D}_{t}^{(j-1) \beta} \operatorname{Res}_{\varphi}(x, t)_{t=0}=\mathcal{D}_{t}^{(j-1) \beta} \operatorname{Res}_{\varphi}^{j}(x, t)_{t=0}$ for each $j=1,2, \ldots, k$.

Anyhow, the next algorithm assists us in determining the required coefficients $\varphi_{n}(x)$, in expansion (3), as well predict and obtain the MFPS solution of FNWSEs (1) and (2): 
Algorithm 1. To find out the coefficients $\varphi_{n}(x)$, in expansion (3), do the following steps:

- $\quad$ Step 1: Assume that the solution of FNWSEs (1) and (2) has the MFPS about $t_{0}=0$ :

$$
\varphi(x, t)=\sum_{n=0}^{\infty} h_{n}(x) \frac{t^{n \beta}}{\Gamma(n \beta+1)},
$$

where $0<\beta \leq 1,(x, t) \in I \times[0, \rho), \rho=R^{1 / \beta}$, and $\varphi(x, t): I \times[0, \rho) \rightarrow \mathbb{R}$ such that $\varphi(x, t)$ is continuous on $I \times[0, \rho), \mathcal{D}_{t}^{k \beta} \varphi(x, t) \in C(0, \rho)$ for $k=1,2, \ldots$.

- $\quad$ Step 2: Define the kth truncated series $\varphi_{k}(x, t)$ of $\varphi(x, t)$ such that

$$
\varphi_{k}(x, t)=\sum_{n=0}^{k} h_{n}(x) \frac{t^{n \beta}}{\Gamma(n \beta+1)} .
$$

- Step 3: Consider the initial condition $\varphi(x, 0)=h_{0}(x)$, then the zeroth MFPS approximate solution of $\varphi(x, t)$ is $\varphi_{k}(x, t)=h_{0}(x)+\sum_{n=1}^{k} h_{n}(x) \frac{t^{n \beta}}{\Gamma(n \beta+1)}$.

- $\quad$ Step 4: Define the kth residual function $\operatorname{Res}_{\varphi}^{k}(x, t)$ such that

$$
\operatorname{Res}_{\varphi}^{k}(x, t)=\mathcal{D}_{t}^{\beta} \varphi_{k}(x, t)-a \mathcal{D}_{x}^{2} \varphi_{k}(x, t)-b \varphi_{k}(x, t)+c \varphi_{k}^{p}(t, x)
$$

- $\quad$ Step 5: Substitute the $k$ th truncated series $\varphi_{k}(x, t)$ into the $k t h$ residual function $\operatorname{Res}_{\varphi}^{k}(x, t)$ such that

$$
\begin{gathered}
\operatorname{Res}_{\varphi}^{k}(x, t)=\mathcal{D}_{t}^{\beta}\left(h_{0}(x)+\sum_{n=1}^{k} h_{n}(x) \frac{t^{n \beta}}{\Gamma(n \beta+1)}\right)-a \mathcal{D}_{x}^{2}\left(h_{0}(x)+\sum_{n=1}^{k} h_{n}(x) \frac{t^{n \beta}}{\Gamma(n \beta+1)}\right)- \\
b\left(h_{0}(x)+\sum_{n=1}^{k} h_{n}(x) \frac{t^{n \beta}}{\Gamma(n \beta+1)}\right)+c\left(h_{0}(x)+\sum_{n=1}^{k} h_{n}(x) \frac{t^{n \beta}}{\Gamma(n \beta+1)}\right)^{p} .
\end{gathered}
$$

- Step 6: Set $k=1$ in Step 5, then by using $\operatorname{Res}_{\varphi}^{1}(x, t)_{t=t_{0}}=0$, the first unknown coefficient $\varphi_{1}(x)$ is obtained. Therefore, the first approximate PS solution $\varphi_{1}(x, t)$ is also obtained.

- $\quad$ Step 7: For $k=2,3, \ldots, m$, do the following subroutine:

(A) Apply the operator $\mathcal{D}_{t}^{\beta},(k-1)$ times, on both sides of the kth residual function $\operatorname{Res}_{\varphi}^{k}(x, t)$ in Step 4 such that $D_{t}^{(n-1) \beta} \operatorname{Res}_{\varphi}^{k}(x, t)$.

(B) Compute the resulting equation at $t=0$ with equality to zero such that $\mathcal{D}_{t}^{(k-1) \beta} \operatorname{Res}_{\varphi}^{k}(x, 0)=0$, with the help of $\mathcal{D}_{t}^{\beta} t^{q}=0$ for $q>\beta$ at $t=0$.

(C) Find the $k$ th unknown coefficient $\varphi_{k}(x)$ and do Step 7 for $k=k+1$ until the arbitrary $m$.

- Step 8: Collect the obtained coefficients $\varphi_{k}(x)$ for each $k=0,1,2, \ldots, m$ in terms of expanded MFPS $\varphi_{k}(x, t)$ and try to find a general pattern with the term of infinite series so that the exact solution $\varphi(x, t)$ of FNWSEs (1) and (2) is obtained; otherwise, the pattern obtained in the sense of the series coefficients will be the mth approximate MFPS solution of FNWSEs (1) and (2).

- $\quad$ Step 9: Stop.

Theorem 3. Let $\varphi(x, t)$ be the exact solutions of FNWSEs (1) and (2). If there exists a fixed constant $\mu \in[0,1]$ such that $\left\|\varphi_{n+1}(x, t)\right\| \leq \mu\left\|\varphi_{n}(x, t)\right\|$ for all $n \in \mathbb{N}$ for all $k \in \mathbb{N}, x \in I$, and $t \in\left[t_{0}, t_{0}+R^{1 / \beta}\right]$, then the sequence of approximate solution converges to $\varphi(x, t)$ as $n \rightarrow \infty$.

Proof. For all $x \in I$ and $t \in\left[t_{0}, t_{0}+R^{1 / \beta}\right]$, let $h_{0}(x)=\varphi(x, 0)=h(x)$, then from $\left\|\varphi_{n+1}(x, t)\right\| \leq \mu \|$ $\varphi_{n}(x, t) \|$, we have $\left\|\varphi_{1}(x, t)\right\| \leq \mu\left\|\varphi_{0}(x, t)\right\|=\mu\left\|h_{0}(x)\right\|=\mu\|h(x)\|$. So, $\left\|\varphi_{1}(x, t)\right\| \leq \mu\|h(x)\|$. Similarly, $\left\|\varphi_{2}(x, t)\right\| \leq \mu\left\|\varphi_{1}(x, t)\right\| \leq \mu \mu\|h(x)\|$, that is, $\left\|\varphi_{2}(x, t)\right\| \leq \mu^{2}\|h(x)\|$. Therefore, $\left\|\varphi_{i}(x, t)\right\| \leq \mu^{i}\|h(x)\|$ and hence $\sum_{i=n+1}^{\infty}\left\|\varphi_{i}(x, t)\right\| \leq \sum_{i=n+1}^{\infty} \mu^{i}\|h(x)\|=\|h(x)\| \sum_{i=n+1}^{\infty} \mu^{i}$. 
Consequently, we have

$$
\begin{gathered}
\left\|\varphi(x, t)-\varphi_{n+1}(x, t)\right\|=\left\|\sum_{i=n+1}^{\infty} \varphi_{i}(x, t)\right\| \\
\leq \sum_{i=n+1}^{\infty}\left\|\varphi_{i}(x, t)\right\| \\
\leq\|h(x)\| \sum_{i=n+1}^{\infty} \mu^{i} \\
=\frac{\mu^{n+1}}{1-\mu}\|h(x)\|,
\end{gathered}
$$

which converges to zero as $n \rightarrow \infty$.

\section{Numerical Results and Discussion}

This section aims to present the implementation of the proposed approach in finding approximate solutions for two FNWSEs in order to show the performance and simplicity of the FRPS method.

Example 1. Consider the following linear fractional Newell-Whitehead-Segel equations [9-11]

$$
\mathcal{D}_{t}^{\beta} \varphi(x, t)=\mathcal{D}_{x}^{2} \varphi(x, t)-2 \varphi(x, t), t \geq 0, x \in \mathbb{R}, 0<\beta \leq 1,
$$

with the initial condition

$$
\varphi(x, 0)=e^{x} .
$$

Using the last description of the FRPS algorithm, starting with $\varphi_{0}(x)=\mathrm{e}^{x}$, then the kth residual function for (9) will be as follows

$$
\operatorname{Res}_{\varphi}^{k}(x, t)=\mathcal{D}_{t}^{\beta} \varphi_{k}(x, t)-\mathcal{D}_{x}^{2} \varphi_{k}(x, t)+2 \varphi_{k}(x, t),
$$

where $\varphi_{k}(x, t)=e^{x}+\sum_{n=1}^{k} h_{n}(x) \frac{t^{n \beta}}{\Gamma(n \beta+1)}$. So, the first residual function can be given as

$$
\operatorname{Res}_{\varphi}^{1}(x, t)=\mathcal{D}_{t}^{\beta} \varphi_{1}(x, t)-\mathcal{D}_{x}^{2} \varphi_{1}(x, t)+2 \varphi_{1}(x, t)=h_{1}(x)+\mathrm{e}^{x}+\left(2 h_{1}(x)-h_{1}^{\prime \prime}(x)\right) \frac{t^{\beta}}{\Gamma(\beta+1)} .
$$

Based on the fact that $\operatorname{Res}_{\varphi}^{1}(x, 0)=0$, the first unknown coefficient of the MFPS expansion (3) is $h_{1}(x)=-\mathrm{e}^{x}$. Thus, the first MFPS approximate solution for (9) and (10) is $\varphi_{1}(x, t)=\mathrm{e}^{x}-\mathrm{e}^{x} \frac{t^{\beta}}{\Gamma(\beta+1)}$.

Similarly, put $k=2$ into Equation (11), then the second residual function will be as

$$
\operatorname{Res}_{\varphi}^{2}(x, t)=\mathcal{D}_{t}^{\beta} \varphi_{2}(x, t)-\mathcal{D}_{x}^{2} \varphi_{2}(x, t)+2 \varphi_{2}(x, t)=\left(h_{2}(x)-\mathrm{e}^{x}\right) \frac{t^{\beta}}{\Gamma(\beta+1)}+\left(2 h_{2}(x)-h_{2}^{\prime \prime}(x)\right) \frac{t^{2 \beta}}{\Gamma(2 \beta+1)},
$$

and the $\beta$ th time fractional derivative of $\operatorname{Res}_{\varphi}^{2}(x, t)$ is $\mathcal{D}_{t}^{\beta} \operatorname{Res}_{\varphi}^{2}(x, t)=\left(h_{2}(x)-\mathrm{e}^{x}\right)+\left(2 h_{2}(x)-h_{2}^{\prime \prime}(x)\right) \frac{t^{\beta}}{\Gamma(\beta+1)}$, then by solving $\mathcal{D}_{t}^{\beta} \operatorname{Res}_{\varphi}^{2}(x, 0)=0$, it yields $h_{2}(x)=\mathrm{e}^{x}$. Hence, the second MFPS approximate solution for (9) and (10) can be written as $\varphi_{2}(x, t)=\mathrm{e}^{x}-\mathrm{e}^{x} \frac{t^{\beta}}{\Gamma(\beta+1)}+\mathrm{e}^{x} \frac{t^{2} \beta}{\Gamma(2 \beta+1)}$.

To find $h_{3}(x)$, write the third truncated series $\varphi_{3}(x, t)=\mathrm{e}^{x}+\sum_{n=1}^{3} h_{n}(x) \frac{t^{n \beta}}{\Gamma(n \beta+1)}$ in Equation (11), taking into account the values of $h_{1}(x)$ and $h_{2}(x)$ in previous steps such that $\operatorname{Res}_{\varphi}^{3}(x, t)=\mathcal{D}_{t}^{\beta} \varphi_{3}(x, t)-\mathcal{D}_{x}^{2} \varphi_{3}(x, t)+$ $2 \varphi_{3}(x, t)=\left(h_{2}(x)-\mathrm{e}^{x}\right) \frac{t^{\beta}}{\Gamma(\beta+1)}+\left(\mathrm{e}^{x}+h_{3}(x)\right) \frac{t^{2 \beta}}{\Gamma(2 \beta+1)}+\left(2 h_{3}(x)-h_{3}^{\prime \prime}(x)\right) \frac{t^{3 \beta}}{\Gamma(3 \beta+1)}$, and through applying $\mathcal{D}_{t}^{2 \beta}$ on the resulting equation, we get $\mathcal{D}_{t}^{2 \beta} \operatorname{Res}_{\varphi}^{3}(x, t)=\left(h_{3}(x)+\mathrm{e}^{x}\right)+\left(2 h_{3}(x)-h_{3}^{\prime \prime}(x)\right) \frac{t^{\beta}}{\Gamma(\beta+1)}$. Then, by utilizing the fact that $\mathcal{D}_{t}^{2 \beta} \operatorname{Res}_{\varphi}^{3}(x, 0)=0$, the coefficient $h_{3}(x)$ will be given such that $h_{3}(x)=-\mathrm{e}^{x}$. Therefore, $\varphi_{3}(x, t)=\mathrm{e}^{x}-\mathrm{e}^{x} \frac{t^{\beta}}{\Gamma(\beta+1)}+\mathrm{e}^{x} \frac{t^{2 \beta}}{\Gamma(2 \beta+1)}-\mathrm{e}^{x} \frac{t^{3 \beta}}{\Gamma(3 \beta+1)}$. 
Using the same manner for $k=4$, and based on the fact that $\mathcal{D}_{t}^{3 \beta} \operatorname{Res}_{\varphi}^{4}(x, 0)=0$, it yields $h_{4}(x)=\mathrm{e}^{x}$. Depending on this, the fourth MFPS approximate solution can be written as $\varphi_{4}(x, t)=\mathrm{e}^{x}-\mathrm{e}^{x} \frac{t^{\beta}}{\Gamma(\beta+1)}+$ $\mathrm{e}^{x} \frac{t^{2 \beta}}{\Gamma(2 \beta+1)}-\mathrm{e}^{x} \frac{t^{3 \beta}}{\Gamma(3 \beta+1)}+\mathrm{e}^{x} \frac{t^{4 \beta}}{\Gamma(4 \beta+1)}$. Moreover, depending on the results of $\mathcal{D}_{t}^{(k-1) \beta} \operatorname{Res}_{\varphi}^{k}(x, 0)=0$, for $k=5,6,7,8$, the eighth MFPS approximate solution for (9) and (10) is given by

$$
\varphi(x, t)=\mathrm{e}^{x}\left(1-\frac{t^{\beta}}{\Gamma(\beta+1)}+\frac{t^{2 \beta}}{\Gamma(2 \beta+1)}-\frac{t^{3 \beta}}{\Gamma(3 \beta+1)}+\frac{t^{4 \beta}}{\Gamma(4 \beta+1)}+\ldots\right) .
$$

Correspondingly, for a particular case, $\beta=1$, the general form of the MFPS approximate solution of (9) and (10) can be written as

$$
\varphi(x, t)=\mathrm{e}^{x}\left(1-\frac{t}{1 !}+\frac{t^{2}}{2 !}-\frac{t^{3}}{3 !}+\frac{t^{4}}{4 !}+\ldots\right)=\mathrm{e}^{x-t},
$$

which coincides with the exact solution of the classical NWSE (9) and (10). Also, it is clear that the obtained results are consistent with those of [9-11].

Figure 1 shows the exact solution and the pattern solution of the approximate solution for different values of $\beta$ where $\beta \in\{0.75,0.85,0.95,1\}$. The analysis of absolute errors by the FRPS algorithm is obtained and summarized in Table 1 for fixed values of $x$ at $n=8$ and at some selected grid points of $t$ with step size 0.16 . Furthermore, the absolute and relative errors associated with the $n$th approximate solutions $\varphi_{n}(x, t)$ when $n=4,6,8$, and $n=12$ for different values of $t$ in $[0,1]$ with step size 0.2 at $\beta=1$ are listed in Table 2. It is obvious from this table that the errors are continuously improved by increasing the number of iterations used, especially at some points near the initial values, where the error is equal to zero after $n=12$. Thus, it can be concluded that increasing the number of FRPS iterations gives higher accuracy of the FRPS method.

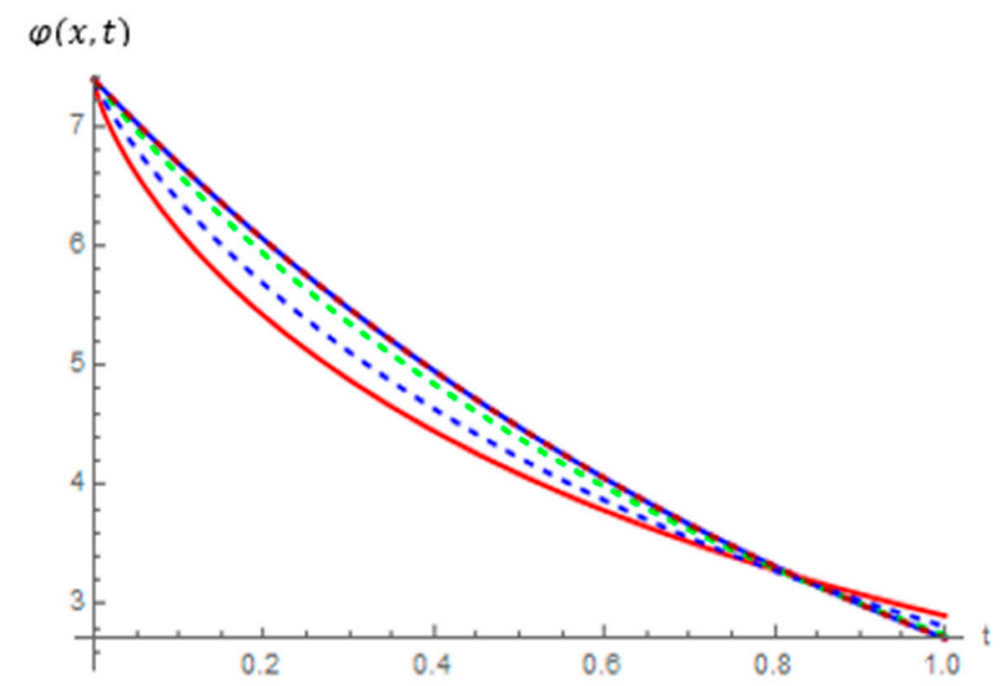

Figure 1. The solution behavior of the approximate solutions $\varphi_{8}(x, t)$ for $\beta \in\{0.75,0.85,0.95,1\}$ compared with the exact solution. Red, $\beta=0.75$; dashed blue, $\beta=0.85$; dashed green, $\beta=0.95$; dashed darker red, $\beta=1$; and blue, exact solution. 
Table 1. Absolute errors of $\varphi(x, t)$ at $\beta=1$ and $n=8$ for Example 1 .

\begin{tabular}{ccccc}
\hline$x$ & $\boldsymbol{t}$ & Exact Solution & Approximation & Absolute Error \\
\hline \multirow{4}{*}{2} & 0.16 & 6.29653826102665 & 6.29653826102803 & $1.377564 \times 10^{-12}$ \\
& 0.32 & 5.36555597112197 & 5.36555597181613 & $6.941567 \times 10^{-10}$ \\
& 0.48 & 4.57222519514215 & 4.57222522141770 & $2.627554 \times 10^{-8}$ \\
& 0.64 & 3.89619330179521 & 3.89619364642941 & $3.446342 \times 10^{-7}$ \\
& 0.80 & 3.32011692273654 & 3.32011945197702 & $2.529240 \times 10^{-6}$ \\
& 0.96 & 2.82921701435156 & 2.82922987150470 & $1.285715 \times 10^{-5}$ \\
\hline & 0.16 & 0.115325121038062 & 0.115325121038087 & $2.524369 \times 10^{-14}$ \\
& 0.32 & 0.098273585604361 & 0.098273585617075 & $1.271391 \times 10^{-11}$ \\
& 0.48 & 0.083743225592195 & 0.083743226073449 & $4.812533 \times 10^{-10}$ \\
& 0.64 & 0.071361269556386 & 0.071361275868581 & $6.312195 \times 10^{-9}$ \\
& 0.80 & 0.060810062625217 & 0.060810108949873 & $4.632465 \times 10^{-8}$ \\
& 0.96 & 0.051818917172725 & 0.051819152659700 & $2.354869 \times 10^{-7}$ \\
\hline
\end{tabular}

Table 2. Error analysis of $\varphi_{n}(x, t), n=4,8,12,16$, at $\beta=1$ for Example 1 .

\begin{tabular}{cccccc}
\hline Iteration & Errors & $\boldsymbol{t}=0.2$ & $t=0.4$ & $t=0.6$ & $t=0.8$ \\
\hline \multirow{2}{*}{$n=4$} & Absolute & $7.013861 \times 10^{-6}$ & $2.173374 \times 10^{-4}$ & $1.599339 \times 10^{-3}$ & $6.535753 \times 10^{-3}$ \\
\cline { 2 - 6 } & Relative & $3.151531 \times 10^{-6}$ & $1.192773 \times 10^{-4}$ & $1.072069 \times 10^{-3}$ & $5.351022 \times 10^{-3}$ \\
\hline \multirow{2}{*}{$n=\mathbf{8}$} & Absolute & $3.759659 \times 10^{-12}$ & $1.887899 \times 10^{-9}$ & $7.119639 \times 10^{-8}$ & $9.304556 \times 10^{-7}$ \\
\cline { 2 - 6 } & Relative & $1.689324 \times 10^{-12}$ & $1.036101 \times 10^{-9}$ & $4.772437 \times 10^{-8}$ & $7.617926 \times 10^{-7}$ \\
\hline \multirow{2}{*}{$n=\mathbf{1 2}$} & Absolute & 0 & $2.88658 \times 10^{-15}$ & $5.46452 \times 10^{-13}$ & $2.26967 \times 10^{-11}$ \\
\cline { 2 - 6 } & Relative & 0 & $1.58419 \times 10^{-15}$ & $3.66298 \times 10^{-13}$ & $1.85825 \times 10^{-11}$ \\
\hline \multirow{2}{*}{$n=\mathbf{1 6}$} & Absolute & 0 & 0 & $8.88178 \times 10^{-16}$ & $5.95364 \times 10^{-16}$ \\
\cline { 2 - 6 } & Relative & 0 & 0 & $6.94999 \times 10^{-14}$ & $1.95599 \times 10^{-12}$ \\
\hline
\end{tabular}

Typically, computer programs have limited precision when viewing data in floating-point format with a fixed number of decimal places. In this sense, if the simulated data is almost identical to the actual data, then the maximum error is around zero. Therefore, in Table 2, we find some absolute errors equal to zero, which indicates that the exact values are very close to approximations at those nodes after a certain number of iterations.

Example 2. Consider the following nonlinear fractional Newell-Whitehead-Segel equation [9-11]

$$
\mathcal{D}_{t}^{\beta} \varphi(x, t)=\mathcal{D}_{x}^{2} \varphi(x, t)+2 \varphi(x, t)-3 \varphi^{2}(x, t), t \geq 0, x \in \mathbb{R}, 0<\beta \leq 1,
$$

with the initial condition

$$
\varphi(x, 0)=\gamma .
$$

To apply the FRPS technique, starting with the initial condition of (17) and based upon (5), the $k$ th MFPS approximate solution of (16) is

$$
\varphi_{k}(x, t)=\gamma+\sum_{n=1}^{k} h_{n}(x) \frac{t^{n \beta}}{\Gamma(n \beta+1)},
$$

where the values of $h_{n}(x), n=2,3, \ldots, k$, can be obtained through construction of $\operatorname{Res}_{\varphi}^{k}(x, t)$ for (16) as follows

$$
\operatorname{Res}_{\varphi}^{k}(x, t)=\mathcal{D}_{t}^{\beta} \varphi_{k}(x, t)-\mathcal{D}_{x}^{2} \varphi_{k}(x, t)-2 \varphi_{k}(x, t)+3 \varphi_{k}^{2}(x, t) .
$$


Now, to determine $\mathrm{h}_{1}(\mathrm{x})$, consider $k=1$ in Equations (18) and (19) so that

$$
\begin{gathered}
\operatorname{Res}_{\varphi}^{1}(x, t)=\mathcal{D}_{t}^{\beta}\left(\gamma+h_{1}(x) \frac{t^{\beta}}{\Gamma(\beta+1)}\right)-h_{1}^{\prime \prime}(x) \frac{t^{\beta}}{\Gamma(\beta+1)}-2\left(\gamma+h_{1}(x) \frac{t^{\beta}}{\Gamma(\beta+1)}\right)-3\left(\gamma+h_{1}(x) \frac{t^{\beta}}{\Gamma(\beta+1)}\right)^{2} \\
=\gamma(3 \gamma-2)+h_{1}(x)+\frac{3 h_{1}^{2}(x) t^{2 \beta}}{\Gamma^{2}(\beta+1)}+\frac{\left((6 \gamma-2) h_{1}(x)-h_{1}^{\prime \prime}(x)\right) t^{\beta}}{\Gamma(\beta+1)} .
\end{gathered}
$$

Then, by using the fact that $\operatorname{Res}_{\varphi}^{1}(x, 0)=0$, it yields $h_{1}(x)=\gamma(2-3 \gamma)$. So, the first MFPS approximate solution of (16) and (17) can be expressed as $\varphi_{1}(x, t)=\gamma+\gamma(2-3 \gamma) \frac{t^{\beta}}{\Gamma(\beta+1)}$.

Again, for finding the second coefficient $h_{2}(x)$, put $k=2$ in Equations (18) and (19), let $h_{1}(x)=$ $\gamma(2-3 \gamma)$, and apply $\mathcal{D}_{t}^{\beta}$ on both sides of the resulting equation as follows

$$
\begin{gathered}
\mathcal{D}_{t}^{\beta} \operatorname{Res}_{\varphi}^{2}(x, t)=h_{2}(x)-h_{1}^{\prime \prime}(x)+\frac{3 \Gamma(\beta+1) h_{1}^{2}(x) t^{\beta}}{\Gamma^{3}(\beta+1)}+\frac{3 \Gamma(4 \beta+1) h_{2}^{2}(x) t^{3 \beta}}{\Gamma^{2}(2 \beta+1) \Gamma(3 \beta+1)} \\
+h_{1}(x)\left(-2+6 \gamma \frac{6 \Gamma(3 \beta+1) h_{2}(x) t^{2 \beta}}{\Gamma(\beta+1) \Gamma^{2}(2 \beta+1)}\right)+\frac{\left((-2+6 \gamma) h_{2}(x)-h_{1}^{\prime \prime}(x)\right) t^{\beta}}{\Gamma(\beta+1)} .
\end{gathered}
$$

Then, by solving $\mathcal{D}_{t}^{\beta} \operatorname{Res}_{\varphi}^{2}(x, 0)=0$, the second coefficient $h_{2}(x)$ will be given such that $h_{2}(x)=\gamma(2-3 \gamma)(2-6 \gamma)$. Hence, $\varphi_{2}(x, t)=\gamma+\gamma(2-3 \gamma) \frac{t^{\beta}}{\Gamma(\beta+1)}+\gamma(2-3 \gamma)(2-6 \gamma) \frac{t^{2 \beta}}{\Gamma(2 \beta+1)}$.

For $k=3$, apply $\mathcal{D}_{t}^{2 \beta}$ on the third residual function of (19), then by solving the resulting fractional equation at $t=0$, one can get $h_{3}(x)=-\frac{3 \Gamma(2 \beta+1) \gamma^{2}(2-3 \gamma)^{2}}{\Gamma^{2}(\beta+1)}+\gamma(2-3 \gamma)(2-6 \gamma)^{2}$. Therefore, the third MFPS will be written as $\varphi_{3}(x, t)=\gamma+\gamma(2-3 \gamma) \frac{t^{\beta}}{\Gamma(\beta+1)}+\gamma(2-3 \gamma)(2-6 \gamma) \frac{t^{2 \beta}}{\Gamma(2 \beta+1)}+$ $\left(-\frac{3 \Gamma(2 \beta+1) \gamma^{2}(2-3 \gamma)^{2}}{\Gamma^{2}(\beta+1)}+\gamma(2-3 \gamma)(2-6 \gamma)^{2}\right) \frac{t^{3 \beta}}{\Gamma(3 \beta+1)}$.

Using the same argument and based upon the result of $\mathcal{D}_{t}^{(k-1) \beta} \operatorname{Res}_{\varphi}^{k}(x, 0)=0$ for $k=4$, the fourth MFPS for nonlinear FNWSEs (16) and (17) can be expressed as:

$$
\begin{gathered}
\varphi_{4}(x, t)=\gamma+\gamma(2-3 \gamma) \frac{t^{\beta}}{\Gamma(\beta+1)}+\gamma(2-3 \gamma)(2-6 \gamma) \frac{t^{2 \beta}}{\Gamma(2 \beta+1)} \\
+\left(-\frac{3 \Gamma(2 \beta+1) \gamma^{2}(2-3 \gamma)^{2}}{\Gamma^{2}(\beta+1)}+\gamma(2-3 \gamma)(2-6 \gamma)^{2}\right) \frac{t^{3 \beta}}{\Gamma(3 \beta+1)} \\
+\left(-\frac{6 \Gamma(3 \beta+1) \gamma^{2}(2-3 \gamma)^{2}(2-6 \gamma)}{\Gamma(\beta+1) \Gamma(2 \beta+1)}+(2-6 \lambda)\left(-\frac{3 \Gamma(2 \beta+1) \gamma^{2}(2-3 \gamma)^{2}}{\Gamma^{2}(\beta+1)}+\gamma(2-3 \gamma)(2-6 \gamma)^{2}\right)\right) \frac{t^{4 \beta}}{\Gamma(4 \beta+1)} .
\end{gathered}
$$

In case $\beta=1$, the approximate solution can be written as

$$
\begin{aligned}
\varphi_{4}(x, t)= & \gamma+\gamma(2-3 \gamma) t+\gamma(2-3 \gamma)(2-6 \gamma) \frac{t^{2}}{2 !}+\left(-18 \gamma^{2}(2-3 \gamma)^{2}+\gamma(2-3 \gamma)(2-6 \gamma)^{2}\right) \frac{t^{3}}{3 !} \\
& +\left(-18 \gamma^{2}(2-3 \gamma)^{2}(2-6 \gamma)+(2-6 \gamma)\left(-6 \gamma^{2}(2-3 \gamma)^{2}+\gamma(2-3 \gamma)(2-6 \gamma)^{2}\right)\right) \frac{t^{4}}{4 !}
\end{aligned}
$$

which coincides with the Taylor series expansion of the exact solution $\varphi(x, t)=\frac{\frac{-2}{3} \gamma e^{2 t}}{\frac{-2}{3}+\gamma-\gamma e^{2 t}}$, as well as being in good agreement with the results obtained in [9-11].

The results obtained by the FRPS method have been drawn in Figure 2 for $x \in[0,1]$ and $t \in[0,0.1]$ at $\beta=1$ and $\gamma=1$. From these figures, it can be noted that the three-dimensional graph of the FRPS approximate solution almost coincides with the behavior of the exact solution considering integer derivatives. The accuracy and efficiency of the FRPS algorithm are shown in Table 3 by computing the fourth FRPS approximate solutions for different cases of $\beta$ at fixed values of $x=0.5$, and $t \in[0,0.1]$, with step size 0.02. For numerical comparison, the proposed method has been compared with the fractional complex transform coupled with He's polynomials (FCT-HP) method [12] in order to verify the superiority of the FRPS method, where the obtained results of Example 2 are listed in Table 4 for $n=5, \beta=1, \gamma=0.001$, and $t \in[0,1]$ with step size 0.16 . Figure 3 shows the behavior of the exact 
solution, fifth FRPS solution and fifth FCT-HP solution [12] at $\gamma \in\{0.05,0.005\}, \beta=1$, and each $t \in[0,1]$, which can illustrate the efficiency of the proposed method. Here, it can be seen that the curve of the FRPS approximate solution is stable and tends to align slightly more to the curve of the exact solution than the fifth FCT-HP solution described in [12] in solving nonlinear FNWSEs (16) and (17). Figure 4 shows the FRPS solution's behavior compared with the behavior of the FCT-HP solution for $n=5$, $x=3$, and $t \in[0,2]$ at different values of fractional order $\beta$ such that $\beta=\{0.45,0.55,0.75,0.85,1\}$. While the surface plots of the fifth-order FRPS solutions for Example 2 at $x \in[-4,4]$ and $t \in[0,2]$ for different values of fractional order $\beta$ such that $\beta=\{1,0.75,0.5,0.25\}$, are given in Figure 5 .

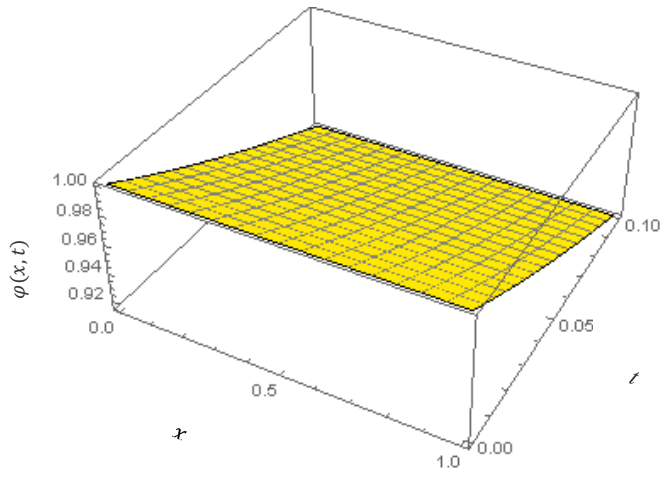

(a)

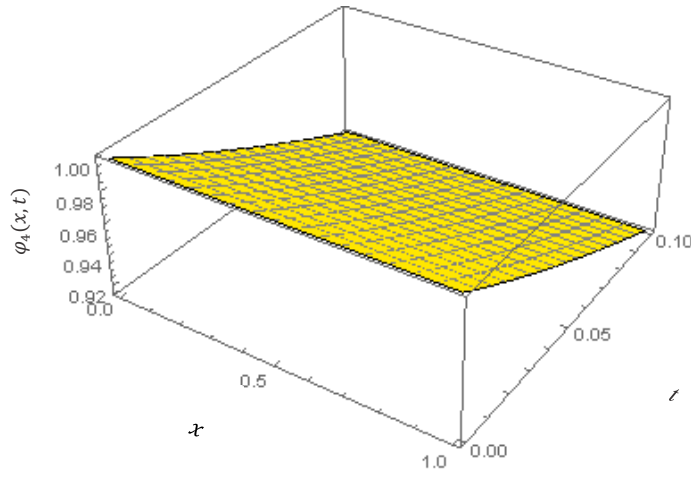

(b)

Figure 2. Solution behavior of the exact $\varphi(x, t)$ and approximate solution $\varphi_{4}(x, t)$ at $\beta=1$, for nonlinear fractional Newell-Whitehead-Segel equations (FNWSEs) (16) and (17): (a) Exact solution; (b) FRPS solution.

Table 3. Approximated solutions of $\varphi(x, t)$ at $n=8$, and $x=0.5$ for Example 2 .

\begin{tabular}{ccccc}
\hline $\boldsymbol{t}_{\boldsymbol{i}}$ & $\boldsymbol{\beta}=1$ & $\boldsymbol{\beta}=0.95$ & $\beta=0.85$ & $\beta=0.75$ \\
\hline 0.01 & 0.99019625 & 0.98749024 & 0.97988049 & 0.96832475 \\
0.02 & 0.98076933 & 0.97640839 & 0.96501163 & 0.94926497 \\
0.03 & 0.97169425 & 0.96609889 & 0.95210517 & 0.93362989 \\
0.04 & 0.96294399 & 0.95638186 & 0.94043021 & 0.91968384 \\
0.05 & 0.95448958 & 0.94714379 & 0.92958436 & 0.90653732 \\
0.06 & 0.94629999 & 0.93829533 & 0.91928645 & 0.89360624 \\
0.07 & 0.93834225 & 0.92975813 & 0.90931428 & 0.88045413 \\
0.08 & 0.93058133 & 0.92145933 & 0.89947874 & 0.86672793 \\
0.09 & 0.92298025 & 0.91332880 & 0.88961102 & 0.85212675 \\
0.10 & 0.91550000 & 0.90529762 & 0.87955555 & 0.83638473 \\
\hline
\end{tabular}

Table 4. Numerical comparison of $\varphi_{5}(x, t)$ at $\beta=1$ and $\gamma=0.001$ for Example 2. FPS, fractional power series; FCT-HP, fractional complex transform coupled with $\mathrm{He}^{\prime}$ s polynomials.

\begin{tabular}{cccccc}
\hline \multirow{2}{*}{$t$} & \multirow{2}{*}{ Exact } & \multicolumn{2}{c}{ FPS Method } & \multicolumn{2}{c}{ FCT-HP Method [12] } \\
\cline { 3 - 6 } & & Fifth Appr. Sol. & Absolute Error & Fifth Appr. Sol. & Absolute Error \\
\hline 0.16 & 0.0013764 & 0.00137634 & $5.54268 \times 10^{-9}$ & 0.00137396 & $2.39074 \times 10^{-6}$ \\
\hline 0.32 & 0.0018939 & 0.00189377 & $1.64202 \times 10^{-7}$ & 0.00187473 & $1.92088 \times 10^{-5}$ \\
\hline 0.48 & 0.0026054 & 0.00260390 & $1.49387 \times 10^{-6}$ & 0.00253977 & $6.56309 \times 10^{-5}$ \\
\hline 0.64 & 0.0035827 & 0.00357487 & $7.81441 \times 10^{-6}$ & 0.00342320 & $1.59483 \times 10^{-4}$ \\
\hline 0.80 & 0.0049238 & 0.00489447 & $2.93697 \times 10^{-5}$ & 0.00459900 & $3.24833 \times 10^{-4}$ \\
\hline 0.96 & 0.0067619 & 0.00667333 & $8.85884 \times 10^{-5}$ & 0.00616420 & $5.97721 \times 10^{-4}$ \\
\hline
\end{tabular}




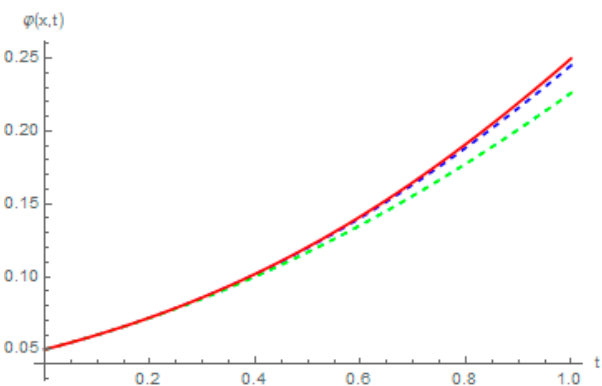

(a)

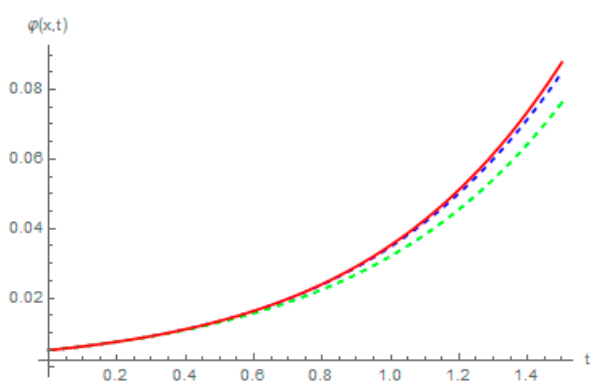

(b)

Figure 3. Plots of the solutions for Example 2 at $\beta=1$ and $t \in[0,1]$. Red for the exact solution; blue for the fractional residual power series (FRPS) solution; green for the FCT-HP solution [12]: (a) Solutions at $\gamma=0.05$; (b) Solution at $\gamma=0.005$.

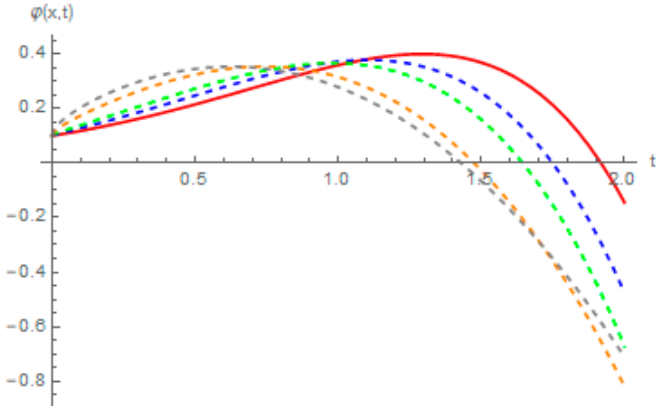

(a)

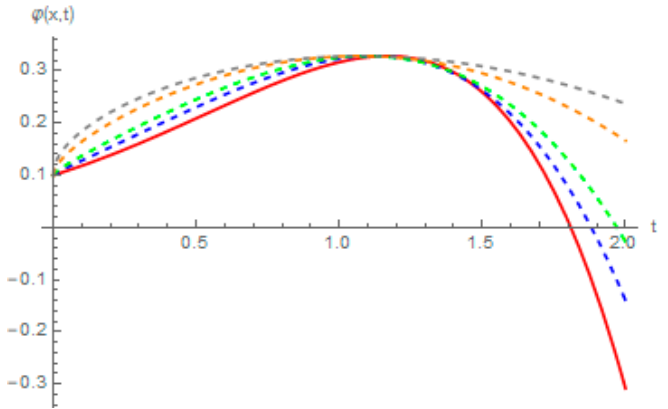

(b)

Figure 4. Plots of the FRPS and FCT-HP solutions for Example 2 at $x=3$ and $t \in[0,2]$ for different values of fractional order $\beta$. Red, $\beta=1$; blue, $\beta=0.85$; green, $\beta=0.75$; orange, $\beta=0.55$; gray, $\beta=0.45$ : (a) FRPS solutions; (b) FCT-HP solutions.

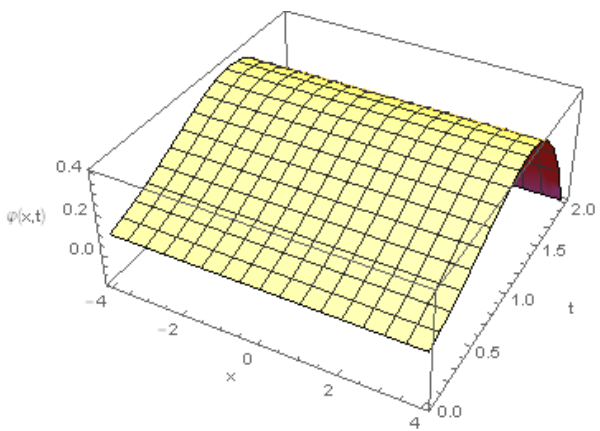

(a)

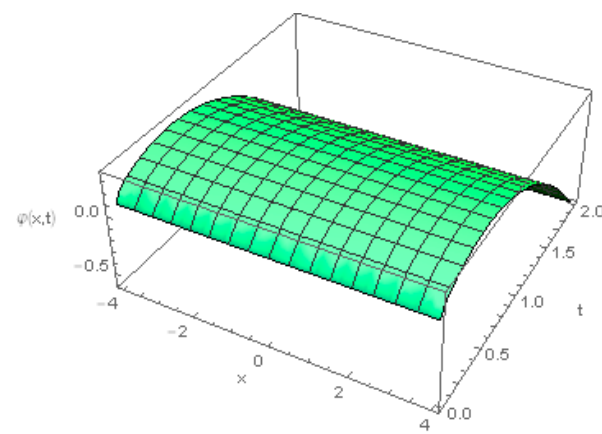

(c)

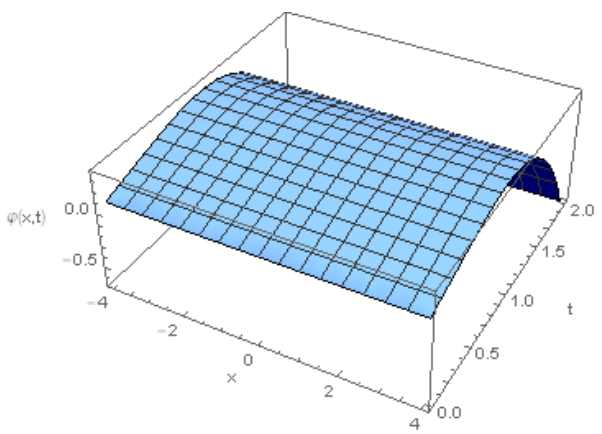

(b)

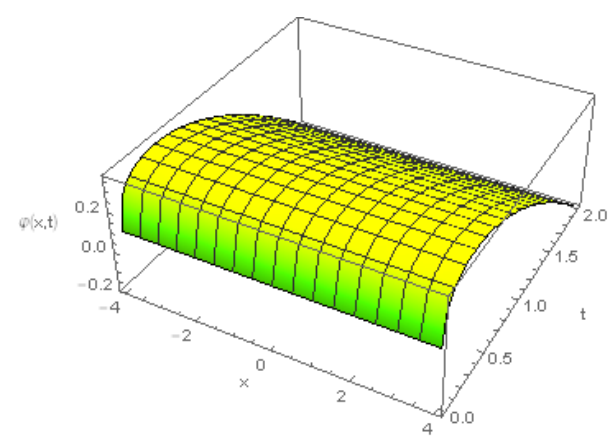

(d)

Figure 5. Surface plots of the fifth-order FRPS solutions for Example 2 at $x \in[-4,4]$ and $t \in[0,2]$ for different values of fractional order $\beta$ such that $\beta=\{1,0.75,0.5,0.25\}$ : (a) $\beta=1$; (b) $\beta=0.75$; (c) $\beta=0.5$; (d) $\beta=0.25$. 


\section{Conclusions}

In this paper, the application of the FRPS algorithm is expanded to explore the approximate solutions of time-fractional Newell-Whitehead-Segel equations in terms of the fractional Caputo derivative. The proposed technique was implemented directly to obtain the approximation solutions in multiple FPS form by deriving the residual error functions. The numerical and graphical results showed coinciding behavior between the analytical solution and the approximate solution of FRPS method at different values of fractional order with a rapid convergence rate even after computing a few iterations. At the same time, these results indicated a good agreement with those obtained by the homotopy analysis sumudu transform method. Moreover, the proposed method does not require any transformation, perturbation, or discretion. From the results, it can be concluded that the FRPS method is a valuable, effective, and straightforward tool in predicting and constructing numeric-analytical solutions for various issues involving fractional partial differential equations. Mathematica 10 software package was used in all computational processes.

Author Contributions: Conceptualization, M.A.; Funding acquisition, R.R.A. and U.K.S.D.; Investigation, R.S.; Methodology, M.A.; Software, M.A.-S.; Supervision, M.A.-S. and R.R.A.; Validation, U.K.S.D.; Visualization, R.S.; Writing—original draft, M.A.; Writing—review and editing, M.A.-S.

Funding: This research was funded by Universiti Kebangsaan Malaysia grant number GP-K007788 and GP-K006926.

Conflicts of Interest: The authors declare no conflict of interest.

\section{References}

1. Miller, K.S.; Ross, B. An Introduction to the Fractional Calculus and Fractional Differential Equations; Wiley: New York, NY, USA, 1993.

2. Baleanu, D.; Machado, J.A.T.; Luo, A.C. Fractional Dynamics and Control; Springer: Berlin/Heidelberg, Germany, 2012.

3. Kilbas, A.A.; Srivastava, H.M.; Trujillo, J.J. Theory and Applications of Fractional Differential Equations, 1st ed.; Elsevier: Amsterdam, The Netherlands, 2010; p. 523. ISBN 0444518320.

4. Oldham, K.B.; Spanier, J. The Fractional Calculus; Academic Press: New York, NY, USA, 1974.

5. El-Ajou, A.; Abu Arqub, O.; Momani, S. Approximate analytical solution of the nonlinear fractional KdV-Burgers equation: A new iterative algorithm. J. Comput. Phys. 2015, 293, 81-95. [CrossRef]

6. Al-Smadi, M. Simplified iterative reproducing kernel method for handling time-fractional BVPs with error estimation. Ain Shams Eng. J. 2018, 9, 2517-2525. [CrossRef]

7. Rosu, H.C.; Cornejo-Perez, O. Super symmetric pairing of kinks for polynomial nonlinearities. Phys. Rev. E 2005, 4, 1-13.

8. He, J.M. Homotopy perturbation technique. Comput. Methods Appl. Mech. Eng. 1999, 178, 257-262. [CrossRef]

9. Kumar, D.; Prakash, R. Numerical approximation of Newell Whitehead-Segel equation of fractional order. Nonlin Eng. 2016, 5, 81-86. [CrossRef]

10. Prakash, A.; Goyal, M.; Gupta, S. Fractional variational iteration method for solving time-fractional Newell-Whitehead- Segel equation. Nonlin Eng. 2019, 8, 164-171. [CrossRef]

11. Prakash, A.; Verma, V. Numerical Method for Fractional Model of Newell-Whitehead-Segel Equation. Frontiers in Physics 2019, 7, 15. [CrossRef]

12. Edeki, S.O.; Ogundile, O.P.; Osoba, B.; Adeyemi, G.A.; Egara, F.O.; Ejoh, A.S. Coupled FCT-HP for Analytical Solutions of the Generalized Time-fractional Newell-Whitehead-Segel Equation. WSEAS Trans. Syst. Control 2018, 13, 266-274.

13. Al-Smadi, M.; Freihat, A.; Khalil, H.; Momani, S.; Khan, R.A. Numerical multistep approach for solving fractional partial differential equations. Int. J. Comput. Methods 2017, 14, 1750029. [CrossRef]

14. Altawallbeh, Z.; Al-Smadi, M.; Komashynska, I.; Ateiwi, A. Numerical solutions of fractional systems of two-point BVPs by using the iterative reproducing kernel algorithm. Ukrainian Math. J. 2018, 70, 687-701. [CrossRef] 
15. Al-Smadi, M.; Abu Arqub, O. Computational algorithm for solving fredholm time-fractional partial integrodifferential equations of dirichlet functions type with error estimates. Appl. Math. Comput. 2019, 342, 280-294. [CrossRef]

16. Al-Smadi, M. Solving fractional system of partial differential equations with parameters derivative by combining the GDTM and RDTM. Nonlinear Stud. 2019, 26, 587-601.

17. Momani, S.; Abu Arqub, O.; Freihat, A.; Al-Smadi, M. Analytical approximations for Fokker-Planck equations of fractional order in multistep schemes. Appl. Comput. Math. 2016, 15, 319-330.

18. Alshammari, S.; Al-Smadi, M.; Hashim, I.; Alias, M.A. Applications of fractional power series approach in solving fractional Volterra integro-differential equations. In AIP Conference Proceedings; AIP Publishing: Melville, NY, USA, 2019; Volume 2111, p. 020003.

19. Kumar, A.; Kumar, S.; Yan, S.P. Residual power series method for fractional diffusion equations. Fundam. Inform. 2017, 151, 213-230. [CrossRef]

20. Zhang, Y.; Kumar, A.; Kumar, S.; Baleanu, D.; Yang, X.J. Residual power series method for time fractional Schrödinger equations. J. Nonlinear Sci. 2016, 9, 5821-5829. [CrossRef]

21. Alaroud, M.; Al-Smadi, M.; Ahmad, R.R.; Din, S.K.U. Numerical computation of fractional Fredholm integro-differential equation of order $2 b$ arising in natural sciences. J. Phys. Conf. Ser. 1212, 2019, 012022.

22. Freihet, A.; Hasan, S.; Al-Smadi, M.; Gaith, M.; Momani, S. Construction of fractional power series solutions to fractional stiff system using residual functions algorithm. Adv. Differ. Equ. 2019, 2019, 95. [CrossRef]

23. Al Shammari, M.; Al-Smadi, M.; Abu Arqub, O.; Hashim, I.; Alias, M.A. Adaptation of residual power series method to solve Fredholm fuzzy integro-differential equations. In AIP Conference Proceedings; AIP Publishing: Melville, NY, USA, 2019; Volume 2111, p. 020002.

24. Alaroud, M.; Al-Smadi, M.; Ahmad, R.R.; Din, S.K.U. Computational optimization of residual power series algorithm for certain classes of fuzzy fractional differential equations. Int. J. Differ. Equ. 2018, 2018, 8686502. [CrossRef]

25. Alshammari, S.; Al-Smadi, M.; Al Shammari, M.; Hashim, I.; Alias, M.A. Advanced analytical treatment of fractional logistic equations based on residual error functions. Int. J. Differ. Equ. 2019, 2019, 7609879. [CrossRef]

26. Alaroud, M.; Al-Smadi, M.; Ahmad, R.R.; Din, S.K.U. An Analytical Numerical Method for Solving Fuzzy Fractional Volterra Integro-Differential Equations. Symmetry 2019, 11, 205. [CrossRef]

27. Freihet, A.; Hasan, S.; Alaroud, M.; Al-Smadi, M.; Ahmad, R.R.; Din, S.K.U. Toward computational algorithm for time-fractional Fokker-Planck models. Adv. Mech. Eng. 2019, 11, 1-11. [CrossRef]

28. Hasan, S.; Al-Smadi, M.; Freihet, A.; Momani, S. Two computational approaches for solving a fractional obstacle system in Hilbert space. Adv. Differ. Equ. 2019, 2019, 55. [CrossRef] 